\title{
Características textiles de la fibra de vicuña (Vicugna vicugna) en el Centro de Investigación, Producción y Transferencia Tecnológica Tullpacancha - Huancavelica
}

\author{
Characteristics of the Textile Fiber of the Vicuna (Vicugna vicugna) \\ at the Research, Production and Technological Transference Center in \\ Tullpacancha - Huancavelica
}

\author{
Juana M. Zavaleta Luján", Lyana Quispe Ochoa ${ }^{* *}$ y Maite Baquerizo Revilla ${ }^{* * *}$.
}

http://dx.doi.org/10.21503/CienciayDesarrollo.2011.v14.04

\section{RESUMEN}

El objetivo del presente estudio fue determinar las características textiles de la fibra de vicuña (Vicugna vicugna) del Centro de Investigación, Producción y Transferencia Tecnológica (CIPTT) Tullpacancha - Huancavelica. La población de vicuñas del CIPTT Tullpacancha fue de 1059 animales en estado de semicautiverio. Se trabajó con 80 muestras de fibra de vicuñas adultas de ambos sexos, escogidas al azar durante el chaccu. Las muestras de fibra fueron de $3 \mathrm{~g}$ de peso, tomadas de la zona del costillar medio, almacenadas y traídas a Lima para su procesamiento en el Laboratorio de Pieles y Curtiembre de Ovinos y Camélidos Americanos (POCA) de la Universidad Nacional Agraria La Molina (UNALM). La finura de fibra se obtuvo por el método Sirolan Laserscan IWTO 98, la longitud de fibra por el peinador Sutter, y el rendimiento al lavado mediante el Leviatan y balanza analítica. Los resultados fueron: diámetro de fibra de vicuñas machos en promedio de 13,62 $\pm 3,14 \mu$, y en hembras de 13,68 $\pm 3,04 \mu$. La longitud de fibra en promedio fue de $0,74 \pm 0,38$ pulgadas en machos, y $0,75 \pm 0,38$ pulgadas para hembras. El rendimiento al lavado fue de $83,81 \%$ en machos y $80,41 \%$ en hembras. Se concluye, así, que la fibra de vicuña del CITTP - Tullpacancha, Huancavelica, presenta valores de $13,62 \pm 3,14 \mu, 0,74 \pm 0,38$ pulgadas y $83,82 \%$ en los machos, y de $13,68 \pm 3,04 \mu, 0,75 \pm 0,38$ pulgadas y $80,42 \%$ en el caso de las hembras, para los tres parámetros evaluados.

Palabras clave: industria textil, longitud de fibra, cerdas, diámetro de fibra, rendimiento al lavado.

\section{ABSTRACT}

The objective of the research was to determine the textile characteristics of the vicuña (Vicugna vicugna) fiber at the Research, Production and Technology Transference Center (CIPTT) Tullpacancha - Huancavelica, The vicunas population of the CIPTT - Tullpacancha was of 1059 animals in the state of semi-captivity.Eighty samples of adult vicuñas of both sexes were chosen at random during the "chaccu". The sample had a $3 \mathrm{~g}$ weight and it was taken from the middle costal portion. They were stored and transported to Lima to be processed in the Laboratory for fur and tanning of ovines an American camelides (POCA) of the Universidad Nacional Agraria La Molina (UNALM). The fineness of the fiber was obtained using the Sirolan Laserscan IWTO 98 method, the length of the fiber by the Sutter comb

* Médica veterinaria graduada en la EAP de Medicina Veterinaria de la UAP.

** Docente de la EAP de Medicina Veterinaria de la UAP.

*** Docente de la EAP de Medicina Veterinaria de la UAP. (maitebaquerizo@yahoo.es) 
and the wash yield by the Leviatan and an analytical scale. The results show: the fiber diameter of male vicuñas an average of $13,62 \pm 3,14 \mu$, and females was 13,68 $\pm 3,04 \mu$. The average length of the fiber was in males $0,74 \pm 0,38$ inches and $0,75 \pm 0,38$ inches in females. The wash yield results show $83,81 \%$ in males and $80,41 \%$ in females. As a conclusion the vicuna fibers at the in Tullpacancha, Huancavelica show values of $13,62 \pm 3,14 \mu ; 0,74 \pm 0,38$ inches and $83,82 \%$ in male and $13,68 \pm 3,04 \mu ; 0,75 \pm 0,38$ inches and $80,42 \%$ for females for the three evaluated parameters.

Key words: textile industry, fiber length, bristle, fiber diameter, wash yield.

\section{INTRODUCCIÓN}

La vicuña (Vicugna vicugna), recurso animal muy importante para las comunidades altoandinas, produce una de las fibras naturales más finas del mundo, cotizada en más de 500 dólares el kilo en el mercado legal(1). El promedio de producción es de $250 \mathrm{~g}$ de fibra fina cada dos años(2). El color del vellón es canela y se distribuye en forma única y uniforme por el cuerpo del animal(3), característica que le permite una perfecta mimetización en el paisaje de las punas altoandinas. El Perú posee 118 391cabezas(4), lo que constituye el $80 \%$ de la población mundial, y el departamento de Ayacucho es el que presenta la mayor población $(34,12 \%)(4)$; sin embargo, el potencial de soportabilidad del pasto andino nos permitiría criar tres millones de cabezas, las que podrían producir $225 \mathrm{TM}$ anuales de fibra fina y cerca de 600000 cueros por año, además de 12000 TM de carne.(2)

Las características tecnológicas de interés de la fibra de vicuña son: el diámetro, la longitud y el rendimiento al lavado(5). En un proceso fabril, es necesario que las fibras tengan requerimientos específicos de diámetro y longitud $(5,6)$.

El objetivo del estudio fue determinar las características textiles de la fibra de vicuna (Vicugna vicugna) proveniente del Centro de Investigación, Producción y Transferencia Tecnológica Tullpacancha - Huancavelica.

\section{MATERIALES Y MÉTODOS}

EL estudio se realizó en el Centro de Investigación, Producción y Transferencia Tecnológica (CIPTT) Tullpacancha, en la provincia de Churcampas, departamento de Huancavelica. La toma de muestra se realizó durante el chaccu en julio del 2009, y el análisis se llevó a cabo en el Laboratorio de Pieles y Curtiembre del Programa de Ovinos y Camélidos Americanos (POCA) de la Universidad Nacional Agraria La Molina (UNALM), en Lima.

La población de vicuñas del CIPTT Tullpacancha es de 1059 cabezas, y se tomaron muestras de fibra de 80 vicuñas adultas, 40 machos y 40 hembras, escogidas al azar durante el chaccu. La muestra fue de un peso aproximado de 3 g cada una, la cual se obtuvo del vellón esquilado de la porción del costillar medio, teniendo en cuenta que el vellón de la vicuña es uniforme y no presenta diferencia significativa, exceptuando cuello y líneas inferiores, como patas. La finura de la fibra se obtuvo por el método Sirolan Laserscan IWTO 98, la longitud de fibra por el peinador Sutter, y para el rendimiento al lavado se utilizó el Leviatan (tipo de lavadora industrial) y una balanza analítica.

\section{RESULTADOS}

El análisis del diámetro de fibra de 40 vicuñas machos dio como resultado un promedio de 


\section{Tabla 1. Características textiles de la fibra de vicuñas del CIPTT Tullpacancha - Huancavelica}

\begin{tabular}{c|c|c|c}
\hline SEXO & $\begin{array}{c}\text { DIÁMETRO DE } \\
\text { FIBRA }(\mu)\end{array}$ & $\begin{array}{c}\text { LONGITUD DE } \\
\text { FIBRA (Puig) }\end{array}$ & $\begin{array}{c}\text { RENDIMIENTO AL } \\
\text { LAVADO }(\%)\end{array}$ \\
\hline MACHOS & $13,62 \pm 3,14^{(*)}$ & $0,74 \pm 0,38^{(1)}$ & 83,81 \\
\hline HEMBRAS & $13,68 \pm 3,04$ & $0,75 \pm 0,38$ & 80,41 \\
\hline
\end{tabular}

$\left(^{*}\right)$ No hay diferencias por sexo

$13,62 \mu \pm 3,14$, C.V. de 23,07 \%; en el caso de las 40 hembras, el promedio fue de 13,68 $\pm 3,04 \mu$, C.V. de $22,23 \%$.

Los datos hallados para la longitud de fibra fue en los machos $0,74 \pm 0,38$ pulgadas, y en las hembras $0,75 \pm 0,38$ pulgadas con C.V. de 51,77 $\%$ y $51,08 \%$ respectivamente.

Se realizó el análisis de rendimiento al lavado del total de las muestras analizadas ( 40 hembras y 40 machos), obteniéndose $83,81 \%$ en machos y $80,41 \%$ en hembras.

\section{DISCUSIÓN}

El diámetro o finura, la característica tecnológica más importante, se mide en micras $(\mu)(7)$ y constituye una característica racial, la misma que puede ser alterada por variaciones climáticas y sobre todo por el factor alimenticio. Controla entre el 70 al $90 \%$ de la habilidad del hilado, y la diferencia es atribuida a la longitud y otras características. Es tal su importancia, que el precio aumenta proporcionalmente en tanto disminuye el diámetro.(8) El diámetro medio es el principal determinante de su precio, debido a su influencia en la capacidad de producción de hilados y en la naturaleza y el tacto-toque de las prendas producidas con ellas(9).

La finura de fibra de vicuña es de 13 y 14 $\mu$ en promedio $(10,11)$, y la fibra procedente del Perú permite predecir propiedades muy favorables respecto a la suavidad de los tejidos(12).

Recuay en el 2006 cita a Pérez, el cual afirma que la extrema finura es el atributo más sobresaliente de la fibra de vicuña. Una real competencia para la vicuña provendría únicamente de la lana de la cabra Cashmere, con un diámetro de fibra entre 15 y $16 \mu$.(12) La fibra de vicuña sometida al análisis microscópico comprueba que su excepcional finura se debe a que el diámetro transversal de la fibra alcanza de 6 a $11 \mu$, con un promedio de $9 \mu .(13)$. Cuanto más finas las fibras, más fino será el hilo procesado; cuanto más delgado sea el hilo, la lana será mejor cotizada por su uniformidad, resistencia, flexibilidad y $\operatorname{suavidad}(14)$. La finura de la fibra y la uniformidad son muy apreciadas en la industria textil, ya que permiten elaborar hilos muy finos, más uniformes y más resistentes(15).

En el estudio, el promedio del diámetro de fibras de vicuñas machos fue de 13,62 $\pm 3,14 \mu$ con un C.V. de 23,07\%, y el de hembras fue de $13,68 \pm 3,04 \mu$ con C.V. de 22,23\%. Los valores obtenidos se encuentran dentro de los rangos publicados por Pumayalla y Santana, de 13 y $14 \mu$ en promedio(12,10). En Bariloche (Argentina) se reportó un diámetro medio $13,8 \mu$ con C.V. de 21,5\%(16); así mismo, en Huancavelica en el 2008 se obtuvo como resultado un diámetro promedio $13,21 \mu$ con C.V. de $8,4 \%$, con un rango entre $10,8 \mu$ y $16 \mu(17)$. 
Teniendo en cuenta que los resultados obtenidos del estudio están dentro del rango de todas las investigaciones, se debe mencionar que ninguna coincide en la diferenciación entre sexos; todas mencionan mayor diámetro en machos que en hembras, como lo indica Díaz. Las hembras, en comparación con los machos, poseen un menor promedio(18), siendo esta variación provocada por la mayor demanda de alimentación durante la preñez y lactación(10). En la investigación no hay diferencias por sexo. Por lo tanto, si la literatura especifica que el diámetro de la fibra está ligada básicamente a la alimentación, debemos recalcar que el chaccu se realizó durante los meses de transición climática, saliendo de una época de altas precipitaciones fluviales para ir a una época seca. Debido a esto, permanecían rezagos de pastos de alta calidad, predisponiendo a una fibra de mayor diámetro, el mismo que dependerá además del índice de gestación y lactación.

La longitud de fibra es la característica que sigue en importancia al diámetro, y ambas determinan las propiedades manufactureras del material textil. la longitud determina si la fibra va a ser sometida al proceso de peinado o cardado. Así, el peinado inglés requiere fibras de más de $2 \frac{1}{2}$ pulgadas $(6,35 \mathrm{~cm})$, y el francés, de más de $1 \frac{1}{2}$ pulgadas $(3,81 \mathrm{~cm})$. Por su parte, al cardado van fibras de menor longitud, como las de $1 \frac{1}{2}$ pulgadas $(3,81 \mathrm{~cm})(19,12)$.

El largo de la fibra de vicuñas oscila entre 4 y $8 \mathrm{~cm}$ en promedio(12). En 1963, se reportó una longitud de fibra de $1 / 2$ a $2 \frac{1}{2}$ pulgadas $(1,27$ a $6,35 \mathrm{~cm})(19)$.

Respecto a la longitud de fibra, en el estudio se obtuvo un promedio de 0,74 pulgadas $(1,88 \mathrm{~cm})$ para machos, con C.V. de 51,77 $\%$, y de 0,75 pulgadas $(1,90 \mathrm{~cm})$ en hembras, con C.V. 51,08\%. Existe, pues, una notoria diferencia con lo expuesto por Vilcanqui, el cual menciona que la longitud de la fibra de vicuña fluctúa entre 2 y $4 \mathrm{~cm}$, con un promedio de 1,29 $\pm 0,49$ pulgadas (15), pero también con lo que afirma Quispe, que reporta una longitud promedio de fibras de vicuñas adultas de 30,88 $\mathrm{mm}(3,08 \mathrm{~cm})$, con C.V. de $21,15 \%$, y cuyas longitudes van de $20,00 \mathrm{~mm}(2 \mathrm{~cm})$ a 50,00 $\mathrm{mm}(5 \mathrm{~cm})(17)$.

La longitud de la fibra está ligada básicamente a la herencia, en un 50 a 30\%, aunque también depende en grado importante de la influencia del medio ambiente. Además, la longitud de la fibra presenta también variaciones en las hembras por el índice de gestación y lactación. Según los resultados del estudio, la fibra de vicuña del CIPTT Tullpacancha resulta muy corta para las especificaciones técnicas de la comercialización y transformación textil, aunque, según lo mencionado por Díaz, se sabe que la fibra de vicuña puede ser procesada en el sistema peinado (tipo algodón) hasta una longitud mínima comprendida entre $1 / 2$ y $1 / 4$ de pulgada $(1,25$ y $0,635 \mathrm{~cm})(18)$.

La corta longitud de las fibras puede deberse al factor humano, expresado básicamente en dos causas. La primera tiene que ver con el momento de la selección de los animales a esquilar, cuando se debe preferir animales que tenga un aproximado de $2,5 \mathrm{~cm}$ de longitud de mecha, verificada utilizando una regla, debido a que la visión y el tacto no permiten una determinación exacta de la misma. La segunda se halla vinculada al momento de la esquila, cuando se practican dos cortes del vellón o se utiliza una esquiladora muy superficial con la idea de no causar heridas y cortes al animal. Se deben determinar, pues, las razones de esta longitud de fibra, a fin de aplicar programas de mejoramiento genético.

El rendimiento, es decir, el porcentaje de la fibra lavada en comparación con la cantidad original sin tratamiento previo, tiene un promedio de $87 \%(7)$, que supera ampliamente 
los rendimientos de la alpaca $(70 \%)$ y del ovino (50\%). Este extremo grado de limpieza tiene una repercusión favorable en el precio, porque de esta manera el comprador paga exactamente por la fibra, y menos por partículas extrañas. Por otro lado, esta es una característica positiva para el lavado, pues no se hace necesario un tratamiento intensivo con sustancias químicas alcalinas, las mismas que incrementan todavía más los inevitables daños que sufre la fibra en los diferentes procesos de transformación(20). En estudios realizados en la zona sur del Perú, se obtuvieron promedios de rendimiento al lavado de fibra de vicuñas de 75,6\%(19) y 87 $\%(21)$.

El rendimiento al lavado de la fibra de vicuña es de $83,82 \%$ y $80,42 \%$ en un grupo de 40 machos y 40 hembras, respectivamente. Estos son valores superiores a los obtenidos por Baquerizo: $72,67 \%$ en promedio (un promedio de $67,88 \%$ en hembras y de 77,46\%(22) en machos). Por otro lado, en el 2000 se reportó un rendimiento al lavado de $70,7 \%$, que resulta relativamente alto en comparación con los obtenidos en otros países, lo que se atribuye a las condiciones ambientales y de manejo(23). Se puede decir, entonces, que la vicuña presenta fibra limpia (con pocas partículas de tierra y escasa grasa) y un rendimiento de la misma de $87 \%$ al lavado(7, $20,21)$.

Los resultados obtenidos en el estudio se encuentran dentro de los parámetros hallados en la bibliografía consultada. Cabe mencionar, sin embargo, que actualmente esos porcentajes han mejorado, encontrándose inclusive en el rango del 90 al 95\%, y pudiendo mejorar ligeramente en Tullpacancha si se evita la contaminación del vellón al momento de la esquila y durante el envellonamiento, haciendo para ello pequeñas correcciones durante el manejo. Con todo, no se debe perder de vista que el medio ambiente y a la topografía pueden haber influido en los valores encontrados.

\section{REFERENCIAS BIBLIOGRÁFICAS}

1. Zúñiga M.A. La vicuña y su manejo técnico. Centro de Investigación y Fondo Editorial de la Universidad Alas Peruanas, Lima, Perú, 2007.

2. Brack A. Conservación de la vicuña en el Perú. Información técnica. Proyecto Especial de Utilización Racional de la Vicuña. Fondo Editorial del Ministerio de Agricultura y Alimentación, Lima, Perú, 1980.

3. Calle R. Producción y mejoramiento de la alpaca. Fondo del Libro del Banco Agrario del Perú, Lima, Perú, 1982.

4. Consejo Nacional de Camélidos Sudamericanos. Oficina de Información Estadística. Censo de camélidossudamericanos. Consejo Nacional de Camélidos Sudamericanos, Lima, Perú, 2000.

5. Carpio M., Santana B. Estudio preliminar de la longitud de análisis cuticular en la fibra de vicuña. Informes de trabajos de investigación en vicuñas. Volumen I. Universidad Nacional Agraria La Molina, Lima, Perú, 1982.

6. Carpio M. Tecnologia de lanas y comercialización. Universidad Nacional Agraria La Molina, Lima, Perú, 1978.

7. Zárate A. Estudio tecnológico de la lana y fibra. Laboratorio de Fibras Naturales, Pieles y Cueros del Programa de Ovinos y Camélidos Americanos. Universidad Nacional Agraria La Molina, Lima, Perú, 1992.

8. Programa de Ovinos y Camélidos Americanos. Primer Curso Nacional para Clasificadores de Lana y Fibra. Universidad Nacional Agraria La Molina, Lima, Perú, 1984.

9. Sacchero D. "Utilización de medidas objetivas en fibras textiles para determinar calidad". En: Simposium Internacional de Biotecnología Aplicada en Camélidos Sudamericanos, 
2008 Nov. 19-21, Huancavelica, Perú, 2008, pp. 37-65.

10. Santana B. Estudio preliminar de la longitud de análisis cuticular en la fibra de vicuña [tesis de pregrado]. Universidad Nacional Agraria La Molina, Lima, Perú, 1978.

11. Pumayalla A., Carpio M. Capacitación de clasificadores de lana y fibra de alpaca. Fondo editorial del Ministerio de Agricultura y Universidad Nacional Agraria La Molina, Lima, Perú, 1972.

12. Recuay K. Principales caracteristicas físicas de la fibra de vicuñas criadas en cercos permanentes [tesis de pregrado]. Universidad Nacional del Centro del Perú, Huancayo, Perú, 2006.

13. Villarroel J. A study of alpaca fibers [tesis doctoral]. The New South Wales University, Australia, 1959.

14. Greanguel. Manejo adaptativo y uso sustentable del guanaco. En: http://www. guenguel.com.ar/guenguel/nueva.htm. Acceso el 10 octubre de 2008.

15. Vilcanqui H. "Características de la fibra de vicuña (Vicugna vicugna)". Rev Mundo Veterinario. 2008; 20 (6):48-53.

16. Sacchero D.M., Mueller J.P. “Determinación de calidad de vellones de doble cobertura tomando al vellón de vicuña (Vicugna vicugna) como ejemplo". Rev Internacional
Agropecuaria. Instituto Nacional Agropecuario, 2005; 34(2): 143-159.

17. Quispe E., Ramos H., Mayhua P., Alfonso L. "Fibre characteristics of vicuña (Vicugna vicugna mensalis)". Rev Small Ruminant Research. 2010; 93(1): 64-66.

18. Carpio M. Camélidos y socioeconomía andina. Producción de rumiantes menores: Alpacas. Editorial Rerumen, Lima, Perú, 1991.

19. Von Bergen W. Wool handbook. Volume I. Editorial Mack Printing Co., New York, USA, 1963.

20. Hofmann R., Otte K., Ponce C., Ríos M. El manejo de la vicuña silvestre. Volumen I. Editorial Sociedad Alemana de Cooperación Técnica (GTZ), Lima, Perú, 1983.

21. Díaz M. Evaluación de algunas características tecnológicas de la fibra de vicuña de primera y segunda esquila en la ECOMUPSA AyasTarma [tesis de pregrado]. Universidad Nacional del Centro del Perú, Huancayo, Perú, 2004.

22. Baquerizo M. Evaluación del diámetro, longitud y rendimiento al lavado de la fibra de vicuña en el Patronato del Parque de las Leyendas [tesis de pregrado]. Universidad Nacional Agraria La Molina, Lima, Perú, 2000.

23. Aliaga J. Separata del curso de producción de ovinos. Universidad Nacional Agraria La Molina, Lima, Perú, 2000. 